\title{
EchoGéo
}

\section{Les « choix scolaires » des parents de classe moyenne et supérieure dans leur contexte}

Une étude de cas menée auprès de parents délégués dans la métropole de Lyon

\section{Maxime Guinepain}

\section{(2) OpenEdition}

Journals

Édition électronique

URL : https://journals.openedition.org/echogeo/21224

DOI : $10.4000 /$ echogeo.21224

ISSN : 1963-1197

Éditeur

Pôle de recherche pour l'organisation et la diffusion de l'information géographique (CNRS UMR 8586)

Référence électronique

Maxime Guinepain, « Les « choix scolaires » des parents de classe moyenne et supérieure dans leur contexte», EchoGéo [En ligne], 55 | 2021, mis en ligne le 17 mai 2021, consulté le 10 août 2021. URL : http://journals.openedition.org/echogeo/21224; DOI : https://doi.org/10.4000/echogeo.21224

Ce document a été généré automatiquement le 10 août 2021.

EchoGéo est mis à disposition selon les termes de la licence Creative Commons Attribution - Pas d'Utilisation Commerciale - Pas de Modification 4.0 International (CC BY-NC-ND) 


\title{
Les « choix scolaires » des parents de classe moyenne et supérieure dans leur contexte
}

Une étude de cas menée auprès de parents délégués dans la métropole de Lyon

\author{
Maxime Guinepain
}

\section{La reproduction sociale et l' " égalité des chances » dans les pratiques de scolarisation}

1 Au fil des années, les parents voient leurs enfants acquérir une autonomie croissante hors de leur supervision directe. Cet apprentissage reste cependant très encadré par la famille (Rivière, 2017a). Celle-ci leur transmet des repères et des normes qui forment leurs pratiques sociales et spatiales. Or, ces repères sont marqués par les rapports qui distinguent et hiérarchisent les classes sociales (que croisent le genre, les appartenances communautaires ou les groupes racisés). Les relations de pouvoir entre groupes leur confèrent une valeur plus ou moins grande dans l'espace social où ils sont mis en jeu, ce qui conduit à parler de «capitaux» culturels, symboliques ou relationnels, qui viennent s'ajouter au capital économique. La notion de « reproduction sociale » issue des travaux de P. Bourdieu et J.-C. Passeron (1970) rend compte de leur transmission.

Celle-ci n'est toutefois ni passive, ni acquise. Même le maintien d'un statut favorisé exige un effort continu et constant pour préserver la distinction qui le soutient (Guibert Lafaye, 2011), dans un contexte où l'école publique en partie démocratisée (Merle, 2020) relaie les normes dominantes auprès de toute la population au nom d'un principe égalitaire : les inégalités qui dépendent de l'arrière-plan familial, mais aussi du lieu de vie et de scolarisation des enfants, y sont considérées inacceptables. Toutefois, dans les faits, ce système obéit plutôt à une logique d'équité qui consiste à présenter à tout·es les élèves les mêmes opportunités - on parle d'« égalité des chances » - sans que 
toutes ne puissent les saisir (Dubet, 2011). L'origine familiale des enfants continue de jouer un rôle décisif (Ben Ayed et Poupeau, 2010), tout comme le lieu et l'établissement de scolarisation, à l'échelle du pays (Broccolichi et al., 2010) comme à celle d'une aire métropolitaine (Oberti, 2007). Les parents conservent ainsi un rôle important, qu'il soit latent, parce que leur position sociale, sinon leur style d'éducation (situé) prépare plus ou moins bien les enfants à répondre aux attendus de l'école (Lareau, 2011; Rivière, 2017b), ou actif, parce qu'ils peuvent déployer de véritables stratégies pour leur assurer les meilleures places possibles - une aptitude que J.-C. François et F. Poupeau (2009) ont baptisée "sens du placement » et qui repose selon certaines chercheur-ses (Blanchard et Cayouette-Remblière, 2011 ; Van Zanten, 2016) sur une forme de " choix scolaire ».

2 Le travail de recherche sur lequel ce texte s'appuie a porté sur des représentantes déléguées de parents d'élèves de collège (enfants âgés de 10 à 15 ans) du secteur public dans l'aire métropolitaine de Lyon ${ }^{1}$. Très largement issue des classes moyenne et supérieure, cette population jouit d'une position sociale favorable tout en pouvant espérer la renforcer. Je propose ici d'en confronter les discours sur les parcours scolaires, rassemblés au cours de dix neuf entretiens menés au printemps 2017, à un travail statistique associant les données publiques du recensement à la sectorisation scolaire en cours cette année-là. La démarche présentée permet de mettre en perspective les «choix scolaires" décrits par les enquêtées avec le profil d'établissements publics de secteur dont les caractéristiques perçues et mesurées, comparées à l'aide d'une analyse factorielle, sont variées. Elle amène à mettre en regard les discours tenus à ce sujet avec les critères les plus souvent discutés dans la littérature (public des établissements, taux de réussite...) et de faire ressortir un aspect souvent mis de côté : les pratiques de scolarisation ne se résument pas à des stratégies engageant l'avenir des enfants. Elles sont des faits sociaux qui se comprennent dans un contexte, social comme géographique : elles engagent aussi le présent des parents.

\section{Un secteur métropolitain fortement ségrégué}

3 Le cadre géographique de l'étude inclut quatre arrondissements de Lyon et cinq communes avoisinantes. Il s'agit d'un environnement urbain dense, où l'offre scolaire est importante : 32 collèges publics se partagent des secteurs préattribués, auxquels il faut ajouter un établissement destiné aux enfants bilingues sans sectorisation et 11 établissements privés de confession catholique ou juive ${ }^{2}$. Le recours à l'enseignement privé est d'ailleurs important à Lyon : environ un tiers des élèves de collège de l'aire d'enquête y étaient scolarisé'es en 2017, pour une moyenne nationale de 21,8 \% en 2019 (DEPP, 2020). Il s'agit enfin d'un espace fortement ségrégué. L'analyse factorielle réalisée à partir des caractéristiques de chaque secteur (voir illustration 1) distingue nettement un secteur central et des quartiers de banlieue défavorisée. Un gradient centre-périphérie se dessine: pour cette raison, on fera référence aux quatre profils identifiés par la Classification Ascendante Hiérarchique en termes de "centre » et de " couronnes ». Pour préserver l'anonymat, les établissements n'y sont pas précisément situés. 
Illustration 1 - Analyse factorielle de chaque secteur de collège : une ségrégation socio-spatiale marquée qui suit un schéma centre-périphérie

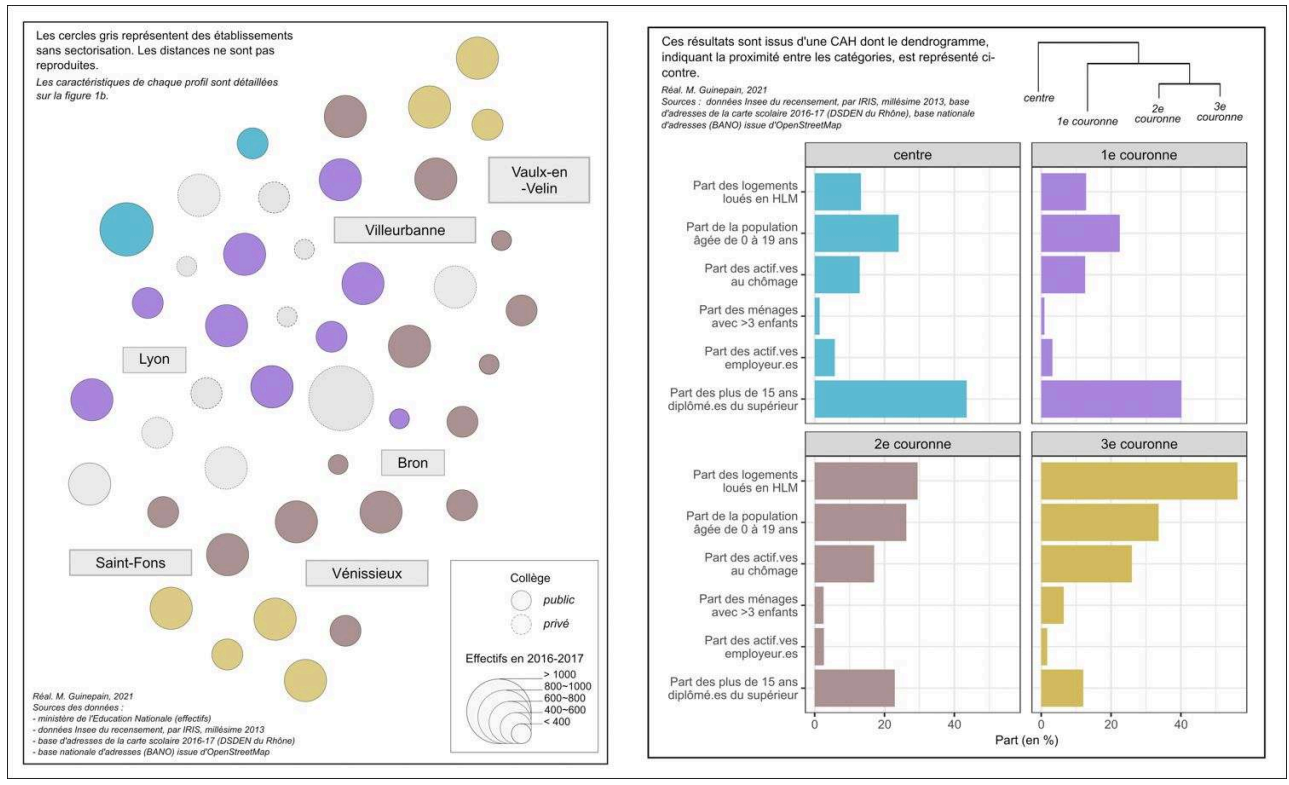

4 En principe, les enfants doivent être scolarisées dans l'établissement desservant leur quartier. Pour chaque niveau, de l'école maternelle au lycée, une grille le désigne en fonction de l'adresse. Ce dispositif datant du milieu des années 1960 est qualifié de « carte scolaire» (Van Zanten et Obin, 2010). Il s'est doté tardivement de l'objectif de limiter la concentration des élèves en réussite, et donc souvent les plus favorisés, dans les établissements les plus cotés : quel que soit leur dossier, ils n'y ont droit qu'en habitant sur leur secteur. À partir de la circulaire ministérielle du 29 décembre 1998, on parle explicitement de "mixité sociale ». La carte scolaire ne fait cependant jamais que se superposer à un espace résidentiel lui-même ségrégué. Sa reconstitution géométrique a bien permis de mettre en évidence que la logique de proximité n'était pas la seule à entrer en compte $(27,1 \%$ des adresses ne sont pas attribuées à l'établissement le plus rapidement accessible à pied), mais le zonage n'atténue que marginalement les contrastes qui s'observent entre les quartiers, et donc les secteurs. L'attribution de résidences de standing récentes à un établissement coté plutôt qu'au plus accessible laisse même à penser que les pouvoirs publics responsables de l'élaboration de la carte scolaire, incluant en l'occurrence la gouvernance métropolitaine, ont parfois d'autres préoccupations.

\section{Malgré la sectorisation, un « quasi-marché scolaire »}

Dans ce cadre, les parents ont malgré tout différents leviers à leur disposition pour inscrire leurs enfants dans un établissement spécifique. Ceux-ci exigent tous l'investissement de ressources inégalement réparties : moyens financiers, mais aussi réseau de connaissances (capital social), disponibilité temporelle pour chercher des informations et aptitude à les trouver, sans parler de l'assurance que les enfants auront le niveau nécessaire pour tirer parti de leur scolarité dans un établissement coté. Pour les parents qui le peuvent, comme c'est le cas de la population enquêtée ici, on pourrait malgré tout parler d'un "quasi-marché scolaire » (Felouzis et al., 2013) où s'exercerait un « choix », terme que l'on discutera plus bas. 
6 Tout d'abord, il faut souligner la continuité qui lie ce «choix» aux mobilités résidentielles. Il est toujours possible de changer d'établissement en changeant de lieu de résidence ; des travaux d'économie ont même montré à Paris que la valeur foncière était affectée par la sectorisation scolaire (Fack et Grenet, 2009). Plusieurs parents témoignent ainsi s'être intéressées de près à la sectorisation lors de leur dernier emménagement; la recherche d'un meilleur environnement intérieur (en termes de confort ou de surface) va de pair avec celle d'un bon environnement extérieur, qui inclut la qualité des écoles, pour les enfants. Cela peut même donner lieu à un arbitrage, comme pour Nathalie (fonctionnaire territoriale, $1^{\text {ère }}$ couronne) :

« Mais en tous cas quand j'ai cherché à m'installer, moi, j'étais tournée... intramuros. [...] [...] Donc c'est vrai que la carte elle joue ; elle joue sur des questions de logement, d'habitat. [...] J'aurais pu avoir, je sais pas moi, 10 à $20 \mathrm{~m}^{2}$ de plus dans des quartiers où je veux plus aller.»

7 Ensuite, la sectorisation officielle peut être contournée de différentes manières. En premier lieu, le secteur privé de l'enseignement, bien qu'il soit en partie subventionné par l'État, n'y est pas soumis. Cette possibilité est si présente à l'esprit des enquêtées que l'une d'elle affirme que le collège public de secteur lui-même est "toujours un

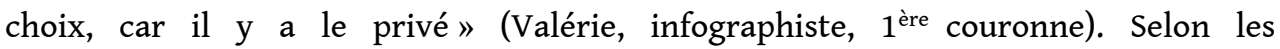
établissements, ce "choix » n'en reste pas moins limité aux familles qui disposent de revenus au moins stables (malgré les aménagements proposés pour les ménages les plus modestes) et aux élèves pouvant présenter de bons dossiers. Par ailleurs, il est possible sous certaines conditions de s'inscrire dans un établissement public hors secteur sur dérogation, sous réserve de pouvoir se justifier d'options ou de besoins spécifiques de l'enfant. Bien qu'elle ait été encouragée en 2007 et qu'elle le soit toujours dans certains quartiers de la $3^{\text {ème }}$ couronne (illustration 1 ) en métropole lyonnaise, cette possibilité semble toutefois fermée pour la plupart des parents au niveau du collège.

Lorsque ces recours sont écartés, certains parents se reportent sur des façons illégales de contourner la carte scolaire, en toute connaissance de cause : "J'ai triché », conclut ainsi Chloé (urbaniste, $1^{\text {ère }}$ couronne). Cela consiste en général à fournir une fausse adresse à l'établissement convoité. Chloé a ainsi joint à son dossier d'inscription un justificatif de domicile maquillé à l'aide d'un outil facilement accessible sur Internet. Auprès des établissements cotés du centre-ville, où les pièces justificatives exigées sont plus nombreuses et mieux contrôlées, des parents peuvent adopter des tactiques bien plus coûteuses. Béatrice (commerçante, centre) témoigne ainsi : « [la principale] nous a parlé des gens qui prennent une location pour quelques mois, qui ne l'utilisent pas forcément, hein ; [...] comme ça, ils ont une adresse sur le secteur. » Ces cas y semblent communs : une déléguée avait obtenu dans ce collège le chiffre de plus de 30 dossiers rejetés pour suspicion de fraude en 2016-17. Sarah (enseignante du primaire, $2^{\text {ème }}$ couronne) et Julia (sans emploi, centre) connaissent des personnes qui ont acquis un studio dans le centre pour pouvoir utiliser son adresse. Cet achat constitue alors un investissement éducatif aussi bien que financier, puisqu'il peut être mis en location. 


\section{Des taux de réussite au hot knowledge : ce qui fait la réputation des établissements}

9 Si le «choix scolaire » s'apparente pour certains parents à une prise de position sur un marché, les modèles économiques supposent qu'ils s'appuient sur un ensemble d'informations pour prendre leur décision (Felouzis et al., 2013). Paradoxalement, car les établissements publics ne sont pas censés être en compétition, celles-ci sont facilement disponibles. On peut mentionner, en premier lieu, l'indicateur phare que constitue le taux d'admission (ou de mentions) au Diplôme National du Brevet, qui est relayé sur de nombreux sites Internet. Sur l'aire d'enquête, ce taux s'échelonnait pour 2016 de 56,9\% (ce qui, à l'échelle nationale, se situe dans le dernier décile) à 93,6\% pour les établissements publics sectorisés, mais il atteint $100 \%$ pour trois des onze établissements privés, ce qui constitue pour eux un argument de "vente» certain. D'autres indices peuvent rendre compte du type d'enseignement dispensé dans un collège (options, presse locale, activités présentées sur les sites académiques...) ou de ses difficultés (classement en Réseau d'Éducation Prioritaire).

10 Toutefois, beaucoup d'enquêtées jugent ces sources trop limitées. Même la bonne volonté dont fait preuve le personnel des établissements de secteur, qui a parfaitement conscience de la situation de concurrence dans lequel ceux-ci sont placés, est souvent interprétée comme le signe que le collège peine à retenir les classes moyennes. Les parents du collège de Sarah (enseignante du primaire, $2^{\text {ème }}$ couronne) ont ainsi été invitées par le principal à y passer quelques heures au printemps précédent, sur le temps de midi, pour en évaluer l'ambiance, et beaucoup n'ont pas été convaincues. Dans l'ensemble, les enquêtées semblent privilégier le "hot knowledge " (Ball et Vincent, 1998), soit une réputation faite de témoignages plus ou moins directs circulant parmi les paires des parents concernés, et inséparable d'un jugement collectif, comme en témoigne Sarah :

« Nous on entendait tout et son contraire, sur le collège, des choses très graves et des choses très bien, donc c'était très difficile de se faire une idée. Durant toute l'année dernière, avec d'autres parents, donc les parents de ses copines principalement, on s'est dits bah voilà : ce serait peut-être pas mal d'y aller ensemble, à ce collège justement... »

11 Enfin, dans la plupart des entretiens, les enquêtées montrent qu'elles et ils s'appuient également sur leurs représentations personnelles des espaces urbains. Comme dans d'autres contextes nationaux plus libéraux (Bunar et Ambrose, 2016), les représentations de la ville et de ses habitantes semblent fixer un premier cadre délimitant ensuite quels seront les espaces pour lesquels les parents mobiliseront des sources plus précises. Toutefois, les propos liés à la composition sociale, voire ethnique, de la population, et donc du public des collèges, restent en général imprécis et nuancés. Les parents enquêtées utilisent pour la plupart une évocation détournée de la qualité (sociale) des populations qu'ils côtoient en empruntant au registre valorisant de la mixité sociale et de la « diversité », tout en dessinant en creux des limites implicites. 


\section{Le « vrai monde » : une mixité valorisée, à condition d'être maîtrisée}

12 Pour bien comprendre le rapport entre les parents enquêtées ici et les milieux qui fréquentent les collèges publics de leurs quartiers, il faut revenir sur leur profil social (voir illustration 2). Tout d'abord, tous les parents ne participent pas aux associations de représentantes. La seule adhésion coûte par exemple $35 €$ pour celle de Khadija (juriste, $2^{\text {ème }}$ couronne), qui reconnaît que cette somme n'est pas anodine pour les familles les plus précaires. Celles-ci y sont, d'un commun accord, largement sousreprésentées. Les personnes qui ont répondu à cette enquête via les associations sont donc celles qui ont le temps et les compétences (professions du social notamment) pour s'engager, et qui ont peut-être estimé avoir une légitimité suffisante pour participer. Presque toutes sont des femmes, ce qui renvoie également aux normes de genre en matière d'implication dans la scolarité des enfants et les sociabilités locales. Par ailleurs, aucune association d'un collège de $3^{\mathrm{e}}$ couronne (illustration 1 ) n'a répondu à mes demandes. Compte tenu de la position sociale des conjointes éventuel.les lorsqu'elle est connue, cet échantillon surreprésente donc très largement la part des classes moyennes et supérieures (une seule enquêtée, qui s'est peu exprimée, exerce une profession manuelle) et se rapproche par ailleurs du pôle « intellectuel » identifié par A. Van Zanten (2016), que la chercheuse distingue d'un pôle «technocrate » dont l'appréhension de l'école serait plus instrumentale.

Illustration 2 - Profil des enquêté es

\begin{tabular}{|l|r|}
\hline Catégorie socio-professionnelle \\
\hline Indépendant.es & 3 \\
\hline Cadre et prof. intellectuelles & 7 \\
\hline Professions intermédiaires & 6 \\
\hline Employé.e & 1 \\
\hline Sans emploi & 2 \\
\hline Inconnue & 6 \\
\hline
\end{tabular}

\begin{tabular}{|l|r|}
\hline Situation d'entretien \\
\hline Seul.e & 11 \\
\hline Présence des enfants & 3 \\
\hline Présence d'autres parents & 2 \\
\hline Invitation à une réunion associative & 2 \\
\hline Total entretiens & 19 \\
\hline
\end{tabular}

\begin{tabular}{|l|r|}
\hline \multicolumn{2}{|l|}{ Typologie du secteur de collège } \\
\hline Centre & 2 \\
\hline $1^{\mathrm{e}}$ couronne & 11 \\
\hline $2^{\mathrm{e}}$ couronne & 10 \\
\hline $3^{\mathrm{e}}$ couronne & 0 \\
\hline Non sectorisé & 2 \\
\hline Genre \\
\hline Femmes & 23 \\
\hline Hommes & 2 \\
\hline Fédération des associations \\
\hline FCPE & 19 \\
\hline PEEP / Assoc. indépendante & 4 \\
\hline Hors association & 2 \\
\hline Total enquêté.es & 25 \\
\hline
\end{tabular}

La présence d'une part significative d'enfants issus des classes populaires dans l'établissement (tout comme dans le quartier ou le voisinage) est dès lors bel et bien vécue par les enquêtées comme une confrontation à l'altérité, dont la manifestation

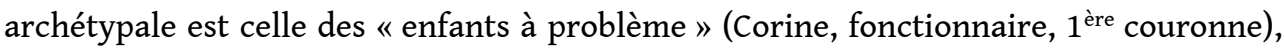
même si cette mise à distance n'est pas forcément dénuée d'empathie; Nathalie (fonctionnaire municipale, $1^{\text {ère }}$ couronne) évoque « des gamins, euh... qui sont vraiment dans de très, très, très grosses difficultés sociales, affectives ». Ce n'est pas uniquement une altérité de classe sociale: au prisme de la "diversité culturelle", certaines enquêtées évoquent des appartenances communautaires, voire une forme de racisation, à l'image d'une mère qui fait écho des difficultés rencontrées par certaines familles sans préciser lesquelles, puis ajoute: «vous imaginez arriver d'un village 
africain ici ? [...] ils sont tout le temps dans la rue chez eux... » ( 1 ère couronne). Le recours au cliché du jeune de banlieue (Hancock, 2008) n'est jamais loin, comme en témoigne la description amusée que plusieurs personnes de secteurs différents donnent de leur établissement, faisant de celui-ci le lieu de réunion des « dealers » et du collège privé le plus proche celui des « acheteurs » de drogue. Conformément à l'analyse d'A. Van Zanten, ces parents «intellectuels» ou "médiateurs" tendent à valoriser cette mixité dont ils reprochent l'absence aux collèges privés. Marine (assistante sociale, $1^{\text {ère }}$ couronne) en fait même un engagement militant :

«Moi, c'était un choix personnel, je défends le public, donc pour moi c'est allé de soi que mes enfants allaient dans cet univers-là, se confronter un peu à tout ce qui existe, et non pas les mettre dans une bulle en choisissant le privé. »

Cette notion de « vraie vie " revient souvent au cours des entretiens et met en lumière des aspects non académiques dans la fréquentation des écoles, qui ont leur importance aux yeux de ce type de parents: les compétences sociales (en l'occurrence, la fréquentation d'enfants différents) font aussi partie de ce qu'on doit y apprendre. Certaines enquêtées insistent également sur l'épanouissement que permet un établissement plus ouvert et moins exigeant envers son public, à l'image de Khadija qui salue l'esprit «soixante-huitard" d'un principal de collège qui "voulait impérativement que le bien-être soit vécu par les enfants et par les parents ».

Cette confrontation à une "mixité" en fait synonyme d'altérité ne se traduit cependant pas par une aspiration à un destin commun avec les enfants des classes populaires. Tout en la valorisant, les enquêtées des $1^{\text {ère }}$ et $2^{\text {ème }}$ couronnes font état de la façon dont le statut de leurs enfants est préservé au sein de l'établissement, en occultant l'aspect social de cette mise à l'écart par un recours à la catégorie de «bon élève ». Cela passe d'abord par l'inscription systématique dans des parcours optionnels d'audience limitée (langues anciennes, allemand, filières bi langues...), dont les parents reconnaissent souvent être à l'origine. La pratique des "classes d'élite " (terme récurrent) est certes officiellement condamnée par les collèges, en particulier dans le contexte de l'éphémère réforme de 2016-17 de l'administration de $\mathrm{N}$. WallaudBelkacem. Les élèves optionnaires sont alors dispersées dans plusieurs classes, mais cela ne les empêche pas de se retrouver lors de voyages à l'étranger qui, comme l'explique Corine (fonctionnaire, $1^{\text {ère }}$ couronne), créent une véritable cohésion de groupe... à condition d'avoir les options dans le cadre desquelles ont lieu ces séjours, ou un bulletin scolaire qui réponde aux exigences pour d'autres sorties présentées comme des récompenses.

17 Ainsi, à l'image de ce qu'avaient montré J.-C. Chamboredon et M. Lemaire (1970), le fait de partager un lieu de vie quelques années tout en restant promises à des trajectoires bien différentes ne gomme pas plus les différences de classe sociale entre les enfants qu'elle ne leur apporte une expérience unique et partagée de l'institution scolaire. Ces murs invisibles sont en partie le fait de parents qui œuvrent activement pour protéger ces trajectoires. 


\section{Choisir pour être un « bon parent » dans les classes moyennes et supérieures}

18 A travers les entretiens menés auprès des parents d'élèves, la notion de "choix " s'avère récurrente et centrale. Largement utilisée dans le langage vernaculaire, elle est reprise par les publications scientifiques qui tentent d'en comprendre les raisons (Van Zanten, 2016), voire de la modéliser sous la forme d'un calcul rationnel, à l'aide de modèles issus du champ de l'économie (Blanchard et Cayouette-Remblière, 2011; Barthon et Monfroy, 2011). Ce recours peut néanmoins être interrogé, sinon, peut-être, déconstruit. Loin d'être neutre, le "choix» fait en effet de plus en plus partie des attendus qui font les «bons parents" (Martin, 2014) : «l'hégémonie du néolibéralisme et la croyance accrue dans la valeur des titres scolaires (credentialism) a amplifié les inquiétudes des parents quant aux conséquences d'une absence de choix éducatifs pour leurs enfants qui pourrait les faire passer pour de "mauvais parents" ", écrivent ainsi D. Reay, G. Crozier et D. James (2013, p. 101) dans un contexte anglo-états-unien où cette politique est encore plus assumée. Cela implique qu'en plus de s'inquiéter du vécu ou de l'avenir de leurs enfants, les parents doivent également se préoccuper de leur propre positionnement social immédiat.

19 Ainsi, ces « choix " sont remis en cause non seulement parce qu'ils peuvent porter à conséquence pour les enfants, mais aussi parce qu'ils sont susceptibles d'être sanctionnés en termes de statut social. Nombreuses sont les mères d'élèves qui évoquent les critiques de leur entourage : pour les amies de Sylvie (chômeuse, $2^{\text {ème }}$ couronne), celle-ci aurait «sacrifié [s]es gamins » en les scolarisant dans le collège de son secteur. L'hostilité générale ressentie à l'encontre des établissements publics explique peut-être qu'en réaction, beaucoup de parents tiennent un discours bien rôdé

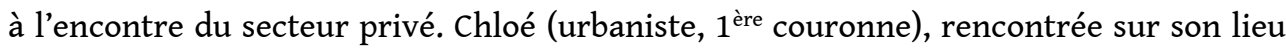
de travail, met même spontanément un débat en place avec une collègue mère d'enfants scolarisés dans un établissement privé voisin: elle devance alors mes questions en énonçant les arguments récurrents sur la primauté du bien-être des enfants ou la «vraie vie ». On comprend en somme que les répondantes ont l'habitude de devoir se justifier de leur « choix » de rester sur le secteur.

Cette mise en scène d'un "choix » volontaire et assumé est de plus accentuée par la situation d'entretien. Les parents mettent d'abord en avant les arguments rationnels et les valeurs qui les ont conduites à privilégier l'inscription sur le secteur, même lorsqu'il s'avère par la suite que ce résultat est le fait de longues hésitations (dont témoignent la précision des renseignements parfois pris sur les établissements privés à portée), de compromis, voire de circonstances fortuites, comme dans le cas de Sarah (enseignante du primaire, $2^{\text {ème }}$ couronne) :

«On a fait nous aussi un dossier pour rentrer dans le privé en se disant bah, on assure nous aussi nos arrières, même si inscrire les enfants dans le privé nous on est contre, par principe [...] mais pour autant notre enfant ne doit pas en pâtir, même si... c'est quand-même assez dingue d'arriver à penser ces choses-là, et puis elle a pas été prise dans le privé, parce qu'il y a eu une histoire de dates [...] et puis ensuite, on s'est dit, euh... Bon, on va quand même y aller dans ce collège... ".

21 L'importance de ces pressions de la part des paires peut en partie expliquer ce qui conduit ces parents à se regrouper dans les associations de parents d'élèves. D'une part, cela leur permet d'exercer une forme de contrôle sur les établissements. L'enjeu en est, 
sinon d'en procéder à la « colonisation » (Van Zanten, 2016), de s'assurer que face aux problèmes rencontrés par les populations précaires, les intérêts des "bons élèves ", c'est-à-dire souvent de leurs propres enfants, restent défendus, en termes d'options proposées par exemple - et de pouvoir en rendre compte. D'autre part, les fédérations réunissent ainsi des parents qui font dans leur entourage la même expérience du dénigrement du secteur public: ils peuvent l'affronter collectivement en portant ensemble un discours de revalorisation et de responsabilité. Enfin, en se confortant dans leur "choix » qui devient collectif (au point que cela soit mis en scène par des enquêtées qui se passent le mot pour participer à mon enquête dans l'un des établissements), ces parents s'assurent du maintien dans leur établissement d'une «masse critique » (Posey-Maddox et al., 2016) de familles de niveau social comparable pouvant assurer que ni leurs enfants, ni elles et eux-mêmes, ne se trouvent isolées.

\section{De l'avenir des enfants au capital social des parents}

Cet engagement collectif permet enfin à certains parents de compenser une position sociale fragilisée, non sans dimension genrée (y compris une forme de solidarité entre femmes). Des enquêtées qui ne travaillent pas ou ont perdu leur emploi peuvent ainsi occuper malgré tout un rôle social valorisant, qui se traduit par un investissement très actif dans les réseaux parentaux, comme l'illustre le cas de Julia (centre). Divorcée au moment de l'enquête, elle n'a jamais occupé d'emploi et dispose de fait de revenus bien inférieurs à ceux de la population aisée qu'elle côtoie, mais dont elle est issue et dont elle maîtrise les codes. Elle peut ainsi prendre place parmi les autres parents comme une égale en investissant un "gros boulot» dans l'association, avec des temps forts fédérateurs qui éprouvent ses compétences de sociabilité et de gestion (ventes au profit de la trésorerie associative par exemple). Dans le contexte d'un secteur populaire en cours de gentrification de la $2^{\text {ème }}$ couronne, Sylvie, au chômage, occupe également un rôle reconnu qui se traduit par un engagement auprès de nombreuses associations. Elle prend l'initiative des prises de parole et mène le débat lors de ma participation à une réunion de représentantes au collège, sans que son statut n'y soit officiel. Sa position la conduit même à se faire porte-parole, en plus de ses propres préoccupations qui ressemblent à celles des autres personnes rencontrées dans le cadre de cette enquête, d'autres parents issus des classes populaires, voire très précaires, et de dénoncer les préjugés dont ils font l'objet.

Plus généralement, les associations de parents d'élèves apparaissent comme des réseaux efficaces pour mettre en relation des personnes qui défendent des intérêts (de classe) communs, se soutiennent et s'engagent, mais aussi pour donner corps aux sociabilités quotidiennes qui constituent une vie de quartier (Authier et al., 2007) centrée sur les écoles et les activités périscolaires de familles qui se ressemblent. De fait, si son appréhension en termes de seul pari sur l'avenir des enfants en rend difficilement compte, le « choix » de l'établissement par ces parents engage aussi leur propre socialisation et la constitution de leur propre entourage dans un collectif que la sectorisation scolaire contribue à ancrer dans un cadre local - ainsi sécurisé pour les plus jeunes : ces deux pans sont inséparables. Cet ancrage se construit en général dans une relative homogénéité sociale, certes atténuée dans le cas des enquêtées les plus investies dans le tissu associatif, même s'il n'est pas anodin qu'elles semblent alors y occuper des rôles plutôt centraux. Le poids de ces «liens faibles» au sens de M. 
Granovetter (1973) gagne ainsi à être pris en compte pour comprendre les pratiques de ces parents de classe moyenne et supérieure face à l'école dans ces contextes urbains ségrégués, en les réinsérant dans un contexte qui leur donne tout leur sens.

\section{Conclusion et discussion}

En les replaçant dans leur contexte local, et social, cet article présente l'hypothèse qu'il est nécessaire de penser les "choix" scolaires des parents de classe moyenne et supérieure comme des processus de décision complexes et encastrés dans les enjeux de positionnement, de reconnaissance et de sociabilités qui les concernent au présent. Cette démarche pourrait permettre de mieux comprendre leurs préoccupations, tout en ne reprenant pas à son compte la responsabilisation individualiste que fait peser sur elles et sur eux l'évaluation de "choix » dont la valeur pourrait être objectivement mesurée, sinon moralement jugée (Martin, 2014). De ce contexte, en l'occurrence, fait partie la posture spécifique du public de cette enquête, majoritairement actif dans les fédérations de parents d'élèves.

Le filtre des parents délégués du centre de la métropole ne permet toutefois pas d'accéder directement à la parole des familles des milieux populaires, qui du fait de leur faible recours à l'évitement scolaire et de leur quasi-absence dans les établissements privés prestigieux, sont rarement étudiées sous l'angle du «choix». C'est une perception que l'on retrouve parmi les enquêtées de cette étude, pour qui les enfants des classes populaires constituent souvent une population peu connue, immuable (ou, dans les collèges cotés du centre-ville, passagère) et silencieuse. Comment ces familles reçoivent-elles des injonctions qui, parce qu'elles sont largement relayées dans les discours politiques et médiatiques, leur parviennent également? Quels mécanismes sociaux produisent et entretiennent leur «non-choix» apparent? Quelles formes d'empowerment envisager dans un cadre où les associations de parents semblent moins actives, ce qui a contribué à les écarter de cette enquête? Ces questions invitent à réfléchir aux modalités qui permettraient de mieux leur donner la parole.

\section{BIBLIOGRAPHIE}

Authier J.-Y., Bacqué M.-H. et Guérin-Pace F., 2007. Le quartier : enjeux scientifiques, actions politiques et pratiques sociales. Paris, Éditions La Découverte.

Ball S.J. et Vincent C., 1998. 'I Heard It on the Grapevine' : "hot" knowledge and school choice. British Journal of Sociology of Education, vol. 19, $n^{\circ}$ 3, p. 377-400.

Barthon C., Monfroy B., 2011. Choix du collège et capital spatial. Espaces populations sociétés, $\mathrm{n}^{\circ}$ 2011/2, p. 321-335.

Ben Ayed C., Poupeau F., 2010. École ségrégative, école reproductive. Actes de la recherche en sciences sociales, $\mathrm{n}^{\circ} 180$, p. 4-10. 
Blanchard M., Cayouette-Remblière J., 2011. Penser les choix scolaires. Revue française de pédagogie. Recherches en éducation, $\mathrm{n}^{\circ} 175$, p. 5-14.

Bourdieu P., Passeron J.-C., 1970. La reproduction. Éléments pour une théorie du système d'enseignement. Paris, Éditions de Minuit, 283 p.

Broccolichi S., Ben Ayed C., Trancart D., 2010. École. Les pièges de la concurrence : comprendre le déclin de l'école française. Paris, Éditions La Découverte, 312 p.

Bunar N., Ambrose A., 2016. Schools, choice and reputation: Local school markets and the distribution of symbolic capital in segregated cities. Research in Comparative and International Education, vol. 11, $\mathrm{n}^{\circ}$ 1, p. 34-51.

DEPP, 2020. Les élèves du second degré à la rentrée 2020. Note d'Information $n^{\circ} 20.37$ [En ligne] URL: https://www.education.gouv.fr/les-eleves-du-second-degre-la-rentree-2020-307010 (consulté le 30 novembre 2020).

Dubet F., 2011. Égalité des places, égalité des chances. Études, n 414, p. 31-41.

Fack G., Grenet J., 2009. Sectorisation des collèges et prix des logements à Paris. Actes de la recherche en sciences sociales, $n^{\circ} 180, p .44$.

Felouzis G., Henriot-Van Zanten A., Maroy C., 2013. Les marchés scolaires: sociologie d'une politique publique d'éducation. Paris, PUF, $217 \mathrm{p}$.

François J.-C., Poupeau F., 2009. Le sens du placement scolaire : la dimension spatiale des inégalités sociales. Revue française de pédagogie. Recherches en éducation, $\mathrm{n}^{\circ}$ 169, p. 77-97.

Granovetter M.S., 1973. The Strength of Weak Ties. American Journal of Sociology, vol. 78, $\mathrm{n}^{\circ}$ 6, p. $1360-1380$.

Guibet Lafaye C., 2011. Le mérite et ses dimensions. In Les Français face aux inégalités et à la justice sociale. Paris, Colin, p. 128-138.

Lareau A., 2011. Unequal childhoods: class, race, and family life, 2. ed., with An update a decade later. Berkeley, University of California Press, $461 \mathrm{p}$.

Martin C. (ed.), 2014. « Être un bon parent » : une injonction contemporaine. Rennes, Presses de l'École des hautes études en santé publique, $246 \mathrm{p}$.

Merle P., 2020. Les inégalités scolaires (1995-2020). Agora débats-jeunesses, nº 86, p. 25-41.

Oberti M., 2007. L'école dans la ville: ségrégation, mixité, carte scolaire. Paris, France, Presses de Sciences Po, 302 p.

Posey-Maddox L., Kimelberg S.M., Cucchiara M., 2016. Seeking a 'critical mass': middle-class parents' collective engagement in city public schooling. British Journal of Sociology of Education, vol. 37, $\mathrm{n}^{\circ}$ 7, p. 905-927.

Reay D., Crozier G., James D., 2013. White middle-class identities and urban schooling, 1. Basingstoke, Palgrave Macmillan, $201 \mathrm{p}$.

Rivière C., 2017a. Du domicile à la ville : étapes et espaces de l'encadrement parental des pratiques urbaines des enfants. Espaces et sociétés, vol. 168-169, n 1, p. 171.

Rivière C., 2017b. La fabrique des dispositions urbaines. Propriétés sociales des parents et socialisation urbaine des enfants. Actes de la recherche en sciences sociales, $\mathrm{n}^{\circ}$ 216-217, p. 64-79.

Van Zanten A., 2016. Choisir son école : stratégies familiales et médiations locales. Paris, Presses Universitaires de France, 283 p. 
Van Zanten A., Obin J.-P., 2010. La carte scolaire. Paris, PUF, 85 p.

\section{NOTES}

1. Quelques parents non délégué·es se sont joint·es à certains entretiens ou m'ont contacté suite à un entretien avec un·e déléguée (illustration 2).

2. La dimension religieuse est variable d'un établissement à l'autre et certains la minorent explicitement au profit d'un discours sur l'ouverture culturelle et l'accueil des « bons élèves ».

\section{RÉSUMÉS}

Cet article repose sur l'association d'une mesure des profils sociaux des secteurs de 32 collèges publics situés dans la métropole de Lyon et d'un ensemble d'entretiens menés auprès de représentant·es de parents d'élèves. Il invite à réinscrire la question du "choix scolaire " des parents de classes moyennes et aisées en milieu urbain ségrégué dans le contexte de leur environnement socio-spatial et montre qu'en plus de l'avenir de leurs enfants, leur propre statut est en jeu.

Focused on the metropolitan area of Lyon (France), this article relies on a comparison between a social profile analysis of 32 state secondary schools' recruitment areas and a series of interviews conducted with parents' representatives. It leads to shed a new light upon the subject of middleand upper-class "school choice" in urban segregated contexts, showing that even beyond their children's future, these parents' own social status is at stake.

\section{INDEX}

Keywords : parent, school map, "school choice", social reproduction, sociability

Mots-clés : carte scolaire, choix scolaire, parent d'élève, reproduction sociale, sociabilité

Thèmes : Sur le Champ - Sur le Terrain

\section{AUTEUR}

\section{MAXIME GUINEPAIN}

Maxime Guinepain, maxime.guinepain@u-paris.fr, est doctorant, rattaché à l'UMR GéographieCités 8504 / UMR ESO 6590. 